\title{
Classifying pulmonary hypertension in the setting of the congenitally malformed heart - cleaning up a dog's dinner
}

\author{
Ingram Schulze-Neick, ${ }^{1}$ Maurice Beghetti ${ }^{2}$ \\ ${ }^{1}$ National UK Service for Pulmonary Hypertension in Children, Cardiac Unit, Great Ormond Street Hospital, \\ London, United Kingdom; ${ }^{2}$ Pediatric Cardiogy Unit, Departement de l'enfant et de l'adolescent, University \\ Hospital of Geneva, Switzerland
}

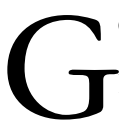
OOD CLASSIFICATIONS OF DISEASES IN HUMANS have contributed greatly to medical progress by improving communication, allowing comparisons, and supporting large scale epidemiologic and therapeutic studies. Well known examples are the classification of malignant tumours, the Diagnostic and Statistical Manual of mental diseases, or the functional classification of exercise tolerance in heart disease prepared by the New York Heart Association, not to mention large works such as the classification of diseases by the World Health Organisation, of which the classification of pulmonary hypertension as a rare, so-called orphan, disease is part.

The advent of targeted specific treatment for pulmonary hypertension in the new millennium made it obvious that a new scheme was needed for the classification of aetiologically different forms so as to reflect their therapeutic accessibility. The revised classification proposed by groups meeting in Evian in $1998,{ }^{1}$ and Venice in $2003,{ }^{2}$ achieved this aim successfully, with many clinical studies based on, and a number of therapeutic substances licensed according to, the new classification.

How was this done? The crucial achievement of the classification has been the fact that a number of diseases are grouped together as "pulmonary arterial hypertension", unifying those forms where the pulmonary vascular disease process takes place in the arterial side of the pulmonary arterial tree and, in most cases, responds to the modern options for treatment provided by agents such as prostanoids,

Correspondence to: Ingram Schulze-Neick, National UK Service for Pulmonary Hypertension in Children, Cardiac Unit, Great Ormond Street Hospital, London WC1N 3JH, United Kingdom. Tel: +44 (0)207 4059200 ext $1005 /$ 1007/6707; Fax: +44 (0)207905 2321; E-mail: neicki@gosh.nhs.uk antagonists of endothelin receptors, and inhibitor of the phosphodiesterase Type V. The new classification also implied similar severity and prognosis in the respective groups of patients, and was used avidly in almost all major therapeutic trials. For example, the idiopathic variant, the old "primary" pulmonary hypertension, and the form associated with congenital cardiac disease, previously considered a "secondary" variant, are probably quite different diseases, despite similarities in features such as the histological aspects. Patients from both of these subgroups, nonetheless, profited from this classification in becoming officially treatable as soon as new substances were licensed for "pulmonary arterial hypertension".

The success of this general classification is in contrast, however, with the still incoherent subclassification of pulmonary arterial hypertension seen in the setting of congenitally malformed hearts, which lacks insight despite notable attempts at improvement, ${ }^{3}$ thus prompting the justified and necessary initiative by van Albada and Berger ${ }^{4}$ to improve the nosology. This is not at all an academic exercise, but a very necessary task in view of the increasing numbers of grown-up patients with congenitally malformed hearts and pulmonary arterial hypertension, who will come to outnumber by far those patients with more "classical" variants of the disease. The difficulties with restructuring the classification, however, soon become apparent. Any modification should be simple enough to allow grouping of sufficient numbers of patients to show efficacy of therapies, but also to satisfy their complicated anatomy and physiology.

Van Albada and Berger ${ }^{4}$ must be commended for their clear analysis of the problems with the current 
Table 1. Existing and suggested classifications of systemic-to-pulmonary shunts associated with pulmonary arterial hypertension (rearranged to allow comparison).

\begin{tabular}{|c|c|c|c|}
\hline Item/Author & WHO (2003) & Galiè (2006) & van Albada \& Berger (2007) \\
\hline 1. type & simple, combined, complex & $\begin{array}{l}\text { pre-tricuspid, post-tricuspid, } \\
\text { combined, complex }\end{array}$ & pre-tricuspid, post-tricuspid \\
\hline 2. dimensions & small, large & $\begin{array}{l}\text { restrictive, non-restrictive, } \\
\text { small, large }\end{array}$ & restrictive, non-restrictive \\
\hline 3. direction of shunt & - & left, right, bidirectional & left, right, bidirectional \\
\hline 4. state of surgical repair & non, partial, complete & non, partial, complete & $\begin{array}{l}\text { non, surgical shunt, pulmonary } \\
\text { artery banding, complete }\end{array}$ \\
\hline 5. associated cardiac anomalies & - & - & $\begin{array}{l}\text { pulmonary arterial stenosis, } \\
\text { pulmonary venous stenosis, } \\
\text { left ventricular dysfunction }\end{array}$ \\
\hline 6. associated extracardiac anomalies & absent/present & absent/present & absent/present \\
\hline
\end{tabular}

classification (See Table 1) by introducing a much needed view, which is the recognition of the haemodynamic burden to the lung. Being led by this variable, they recognise several risk factors for developing pulmonary arterial hypertension, rephrased by us as:

- Pre- or post-tricuspid location of the shunt, in other words with or without additional stress of pressure on the pulmonary arterial system

- Increased flow of blood to the lungs

- Non-restrictive nature of shunting, either in the absence of surgery, or because of a residual lesion despite surgery

- The timing of surgical correction

- The presence of intra- and extracardiac abnormalities

- Individual susceptibility and genetic factors, such as trisomy 21 .

The classification proposed by van Albada and Berger ${ }^{4}$ is certainly useful, and represents a muchneeded step in the right direction. This is because it follows one important rule. Classifications are most useful if they are unidimensional, in other words organised along one easily recognisable variable to form the index, such as age, size, gender, or severity. The more this variable correlates with others, the more does the classification itself assume predictive power. A well-known and successful classification of this type is the one proposed by the New York Heart Association for classification of exercise tolerance. If other variables are introduced to see what else the classification could do, however, in this case to improve understanding of prognosis and therapeutic decisions, as well as to help in the adequate design and interpretation of clinical research, then this introduces the same potential caveats from which the previous classifications have suffered, namely a multidimensional approach which immediately confuses the situation. The reason for this is because the patients not only have pulmonary arterial hypertension. First and foremost they have congenitally malformed hearts.

Neither anatomy alone, nor the haemodynamic risk factors, clearly predict the clinical course and prognosis of pulmonary arterial hypertension as seen in the setting of the congenitally malformed heart. These features vary markedly, depending on institution and doctor providing treatment, as well as the patient him or herself. It is necessary to take all these aspects into account when deciding optimal therapeutic strategies. Treatment of the congenital malformation itself, by surgically normalising or ameliorating circulation and myocardial loading conditions, by corrective or palliative surgery, or even by abstinence from surgery, creates a range of different physiologies. Indeed, these are the issues which interfere with the presently existing systems for classification. This is because the different cardiovascular physiologies that result from corrected or uncorrected malformations may dominate the clinical state and fate of the patient and the response to therapy to a more important degree than the concomitant pulmonary arterial hypertension alone.

What are these special physiologies? This is exactly the issue addressed by van Albada and Berger, ${ }^{4}$ which, if we may take it a few steps further, leads to the following view: first, there is the group of patients that simply needs surgery as opposed to those patients who do not yet have the typical physiology of the Eisenmenger patient, but may still be operable if their pulmonary vascular resistance was minimally lower. This subgroup is actually very important, since the pulmonary vascular disease may progress despite successful surgery. Then, second, there are those patients with haemodynamics similar to idiopathic pulmonary hypertension, namely those with insignificant shunting who have not undergone operative correction, 
or those who have undergone repair but still have insignificant residual defects. There is then a third group of patients in whom the arterial side of the pulmonary vascular tree has developed additional active disease as a reaction to pulmonary venous hypertension which existed either in the past (eg., obstructive lesions on the left side of the heart which with successful repair) or which is an ongoing problem (eg., left myocardial disease with increased enddiastolic pressures). The Eisenmenger physiology makes up a fourth entity with its own important pathophysiological considerations. Those with the Fontan circulation then represent a fifth subset, with very fragile and specific physiology. In these patients, as with those in the other groups, the increase in pulmonary vascular resistance leads ultimately to failure of the circulation. Making up a sixth group, there are those with the unusual forms of congenital malformations that produce unilateral pulmonary arterial hypertension, mainly because of the surgical shunts, systemic-to-pulmonary collateral arteries, abnormalities of the right or left pulmonary arteries. A final seventh group is constituted by those with increased pulmonary vascular resistance due to general hypoplasia of the pulmonary vascular bed, in whom pulmonary vasodilating therapy may be explored in the absence of any other therapeutic options. To make things more complicated, all groups come combined not only with their own past or ongoing risk of developing pulmonary arterial hypertension, but also with the risk of failure of the subpulmonary ventricle to sustain this afterload. Thus classifying these groups according to their underlying physiology (Table 2) shows their common elements determining clinical course and the potential for discussions regarding possibilities of treatment.

The current body of evidence as condensed by van Albada and Berger ${ }^{4}$ regarding the specific treatment of patients with the Eisenmenger syndrome underlines the fact that this new pathophysiologically oriented concept for classification of pulmonary arterial hypertension as seen in the setting of the congenitally malformed heart, shown here with its entire spectrum, is already implicitly used in a fruitful way. This suggested classification, at first glance, may appear to be out of line with the classifications proposed in Evian and Venice, as the group with the Fontan-like circulations does not represent patients with pulmonary hypertension according to the classical and haemodynamically simple definition of an increased pulmonary vascular resistance of greater than 3 Wood units per meter squared, or a mean pulmonary arterial pressure of more than 25 millimetres of mercury. When examined more closely, however, it becomes apparent that this group unequivocally needs to be

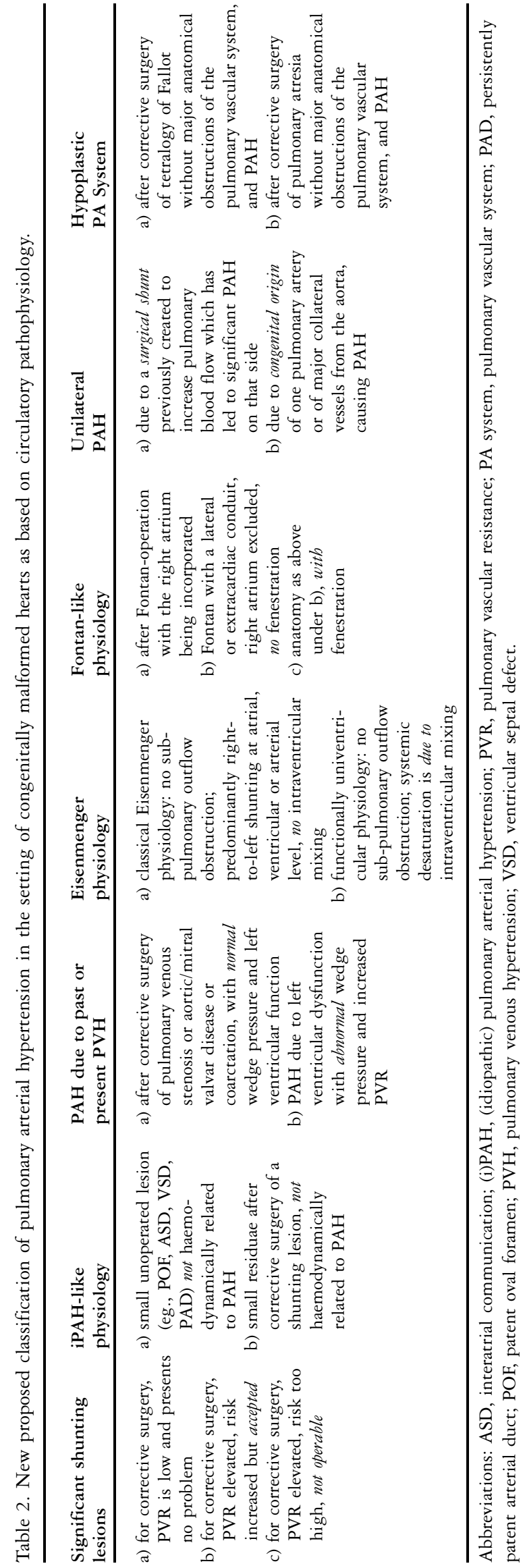


part of this classification, as even a minimal increase in pulmonary vascular resistance causes an important, clinically relevant, problem which is very analogous physiologically with that of patients with classical severe pulmonary arterial hypertension and which results in similar consequences, both in symptoms and most likely necessary medical therapy.

It is clear that, in all classifications, there is and must be an element of simplification, which also implies a specific bias and some weaknesses. A classification, nonetheless, is an instrument with which to work. Thus, it needs to stand the test of time. If successful, it may prove a very useful tool for research, clinical use and ultimately the benefit of patients. Eventually, it will find its place in official recommendations, such as guidelines for treatment. Modifications such as those proposed by van Albada and Berger ${ }^{4}$ are an important and necessary part of this comprehensive process of classification. The next World Congress on Pulmonary Hypertension, to be held in the United States of America in 2008, will develop still another subclassification of pulmonary arterial hypertension as seen in the setting of the congenitally malformed heart, and will aim to improve further the assessment and treatment of these complicated patients. Hopefully those preparing the next modification will take note both of the suggestion of van Albada and Berger, ${ }^{4}$ and our own comments.

\section{References}

1. Rich S. Executive summary from the World Symposium on Primary Pulmonary Hypertension. World Symposium on Primary Pulmonary Hypertension. Evian, France, 1998.

2. Simonneau G, Galie N, Rubin LJ, et al. Clinical classification of pulmonary hypertension. J Am Coll Cardiol 2004; 43: 5S-12S.

3. Galiè N. Classification of patients with congenital systemicto-pulmonary shunts associated with pulmonary arterial hypertension: current status and future directions. In: Beghetti M, Barst R, Naeije R, Rubin L (eds). Pulmonary Arterial Hypertension Related to Congenital Heart Disease. Urban \& Fischer, München Jena, 2006, pp. 11-16.

4. van Albada MI, Berger RMF. Pulmonary arterial hypertension in congenital cardiac disease - the need for refinement of the EvianVenice classification. Cardiol Young 2008; 18. This issue.

5. Schulze-Neick I. Pulmonary hypertension in congenital heart disease. Deutsche Medizinische Wochenschrift (1946) 2006; 131: S322-S324. 